\title{
ON STABILITY OF THE FLOW OF A STRATIFIED GAS OVER A LIQUID*
}

\author{
BY \\ JOHN F. SONTOWSKI ${ }^{1}$, BARRY S. SEIDEL AND WILLIAM F. AMES² \\ University of Delaware
}

Summary. Instabilities of the superposed flow of a gas over a liquid are considered under the assumption of inviscid, incompressible flow. The effects of density stratification in the gas are examined, and in so doing, two separate and different types of instabilities are revealed. As the velocity of the gas relative to the liquid increases from zero, there first appears an instability of a selective and relatively weak nature referred to as the initial instability. This is followed, at higher velocities, by a stronger type of instability called the gross instability. The initial instability takes the form of two distinct waves of different lengths, one superimposed upon the other. This superposition of two waves at low velocity is in accord with experimental observations, as are the calculated critical velocity and wavelength at which they first occur. The gross instability, on the other hand, is composed of a continuous spectrum of unstable waves, and is simply a slight refinement of the classical result of Kelvin. Such an occurence of two separate instabilities is in agreement with Munk's experimentally based contention that Kelvin's solution is not incorrect, as originally believed, but rather represents an actual instability which, however, is preceded by an additional and different type of instability.

1. Introduction. The stability of a superposed flow of two fluids was studied by Kelvin. Considering two different fluids, each having uniform density and velocity distributions and flowing parallel to each other in a horizontal direction with an interface of arbitrary surface tension, Kelvin determined a stability criterion in terms of the relative velocity between the two fluids. Application of this result to the special case of air blowing over water yields a critical velocity of 15 m.p.h. at which instability first occurs. This result has met with general dissatisfaction, since disturbances actually arise on large bodies of water at much lower values of the wind velocity. However, Munk [3] expressed the belief that although not coincident with the onset of instability, the value obtained by Kelvin is indeed a critical value as it marks the occurrence of several other phenomena, such as sudden increases in evaporation, convection and the number of breaking waves. Whether true or not, this does not explain instabilities observed at velocities below Kelvin's critical value. A study of these questions is presented here. For greater detail of the analysis involved, the reader is referred to Sontowski [5].

The flow configuration and assumptions of Kelvin are reexamined here along with the additional consideration of density stratification in the upper fluid. This density

\footnotetext{
*Received April 15, 1968.

'Present address: General Electric Company, King of Prussia, Pennsylvania.

${ }^{2}$ Present address: University of Iowa.
} 


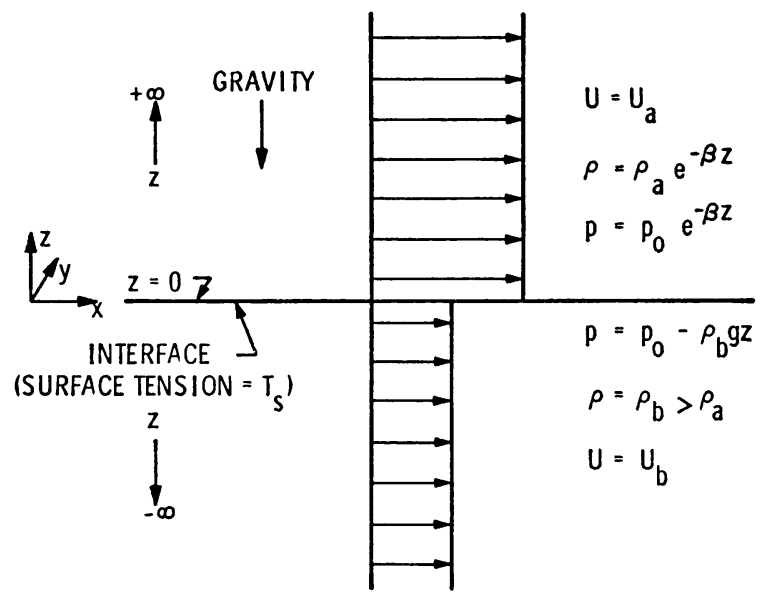

FIg. 1. The assumed stationary state.

stratification will be modeled by an exponentially decreasing function of height. Fig. 1 illustrates the problem.

The fluid velocities to be considered are low, and so the assumption of incompressible flow is reasonable. Viscosity can be neglected by reasoning in terms of the types of instabilities expected in the unbounded flow of a gas over a liquid. In such a situation both the Tollmien-Schlichting and the Kelvin-Helmholtz instabilities may occur. The effect of viscosity is to directly cause instabilities of the Tollmien-Schlichting type and to retard Kelvin-Helmholtz instabilities by moderating the kinetic energy distribution. Thus, by neglecting viscosity the Tollmien-Schlichting instabilities are not expected to appear in the analysis, while the Kelvin-Helmholtz instabilities should occur, but at a velocity lower than reality. Since it is further expected (see Ostrach and Koestel [4]) that Kelvin-Helmholtz instabilities actually occur before TollmienSchlichting instabilities, the assumption of inviscid flow should provide a stability criterion in terms of a velocity difference which somewhat underestimates reality and thus serves as a conservative estimate of the actual inception of instability.

2. Mathematical statement of the problem. Consider two inviscid fluids in a stationary state of horizontal streaming in the $x$-direction and superimpose a disturbance upon this state. Assuming the disturbances to be small and neglecting higher order terms, six linearized perturbation equations in six unknowns are obtained as a result of the requirements of continuity, incompressibility, momentum and interface kinematics. The method of normal modes is now employed. Seeking solutions which depend on $x, y$ and $t$ in the fashion

$$
\exp \left[i\left(k_{x} x+k_{y} y+n t\right)\right]
$$

and substituting into the perturbation equations yields six equations which by a process of elimination are reduced to one equation in one unknown. This equation is 


$$
\begin{aligned}
\left(n+k_{x} U\right)\left(\frac{d^{2}}{d z^{2}}-k^{2}\right) w-k_{x} \frac{d^{2} U}{d z^{2}} w- & \frac{g k^{2}}{\rho} \frac{d \rho}{d z} \frac{w}{n+k_{x} U} \\
& +\frac{1}{\rho} \frac{d \rho}{d z}\left[\left(n+k_{x} U\right) \frac{d w}{d z}-k_{x} \frac{d U}{d z} w\right]=0
\end{aligned}
$$

where $w$ depends only on $z$ and the product $w \exp \left[i\left(k_{x} x+k_{y} y+n t\right)\right]$ is the perturbation of the $z$-component of velocity. The wave number $k$ is defined to equal $\left(k_{x}^{2}+k_{y}^{2}\right)^{1 / 2}$. In addition it is required that the quantity $w /\left(n+k_{x} U\right)$ be continuous across the interface and also that

$$
\Delta_{\bullet}\left[\rho\left(n+k_{x} U\right) \frac{d w}{d z}-\rho k_{x} \frac{d U}{d z} w\right]=g k^{2}\left[\Delta_{\bullet}(\rho)-\frac{k^{2}}{g} T_{\bullet}\right]\left(\frac{w}{n+k_{x} U}\right) .
$$

where $\Delta_{\mathrm{s}}(f)=\lim _{\epsilon \rightarrow 0}\left[f_{z=z_{0}+\epsilon}-f_{\left.z=z_{\mathrm{a}}-\epsilon\right]}\right]$ and $z_{\mathrm{s}}$ represents the undisturbed interface position. Details of the development of these results are given by Chandrasekhar [1, Sec. 100].

Application of the above mathematics to the case described by Fig. 1 requires solutions of the equations

$$
\begin{gathered}
\frac{d^{2} w}{d z^{2}}-\beta \frac{d w}{d z}-k^{2}\left[1-\frac{\beta g}{\left(n+k_{x} U_{a}\right)^{2}}\right] w=0 \quad z>0 \\
d^{2} w / d z^{2}-k^{2} w=0 \quad z<0 .
\end{gathered}
$$

The general solutions are

$$
\begin{array}{ll}
w=A_{a} e^{m+z}+B_{a} e^{m-z} & z>0, \\
w=A_{b} e^{k z}+B_{b} e^{-k z} & z<0
\end{array}
$$

where $A_{a}, B_{a}, A_{b}$ and $B_{b}$ are arbitrary constants and

$$
m_{ \pm}=\frac{\beta}{2} \pm\left[\left(\frac{\beta}{2}\right)^{2}+k^{2}\left[1-\frac{g \beta}{\left(n+k_{x} U_{a}\right)^{2}}\right]\right]^{1 / 2} .
$$

Boundary conditions disallow disturbances which increase exponentially as the outer bounds of the fluids are approached. Thus

$$
\begin{array}{ll}
w=B_{a} e^{m_{-} s} & z>0, \\
w=A_{b} e^{k s} & z<0
\end{array}
$$

with the requirement that

$$
\operatorname{Re}\left[\left(\frac{\beta}{2}\right)^{2}+k^{2}\left[1-\frac{g \beta}{\left(n+k_{x} U_{a}\right)^{2}}\right]\right]^{1 / 2} \geq \frac{\beta}{2} .
$$

Continuity of $w /\left(n+k_{x} U\right)$ across the interface leads to the following solution in terms of one arbitrary constant $A$ :

$$
\begin{array}{ll}
w=A\left(n+k_{x} U_{a}\right) e^{m-} z & z>0, \\
w=A\left(n+k_{x} U_{b}\right) e^{k z} & z<0 .
\end{array}
$$

Substitution into the second interface condition (3) yields the eigenvalue equation. This equation may be written in dimensionless form as 


$$
\begin{aligned}
\rho_{*} \beta_{k}\left(\nu+k_{x} \bar{U}_{a}\right)^{2}-K\left(\nu+k_{*} \bar{U}_{b}\right)^{2}+\left[\left(1-\rho_{*}\right)+\sigma_{k}\right] & \\
& =\rho_{*}\left(\nu+k_{*} \bar{U}_{a}\right)^{2}\left[1-\frac{2 \beta_{k}}{\left(\nu+k_{*} \bar{U}_{a}\right)^{2}}\right]^{1 / 2}
\end{aligned}
$$

in terms of the eigenvalue $\nu$. Adding the requirement

$$
\operatorname{Re}\left[1-\frac{2 \beta_{k}}{\left(\nu+k_{*} \bar{U}_{a}\right)^{2}}\right]^{1 / 2} \geq \beta_{k}
$$

completes the specification of the eigenvalue problem. According to definition

$$
\begin{array}{rlrl}
\nu & =\frac{\left[\left(\frac{\beta}{2}\right)^{2}+k^{2}\right]^{1 / 4}}{k g^{1 / 2}} n & k_{*}=\frac{k_{x}}{k} & \rho_{*}=\frac{\rho_{a}}{\rho_{b}}, \\
\beta_{k} & =\frac{\frac{\beta}{2}}{\left[\left(\frac{\beta}{2}\right)^{2}+k^{2}\right]^{1 / 2}} \quad K=\frac{k}{\left[\left(\frac{\beta}{2}\right)^{2}+k^{2}\right]^{1 / 2}} \quad \sigma_{k}=\frac{T}{g \rho_{b}} k^{2}, \\
\bar{U}_{a} & =\frac{\left(\left(\frac{\beta}{2}\right)^{2}+k^{2}\right)^{1 / 4}}{g^{1 / 2}} U_{a} & \bar{U}_{b}=\frac{\left(\left(\frac{\beta}{2}\right)^{2}+k^{2}\right)^{1 / 4}}{g^{1 / 2}} U_{b} .
\end{array}
$$

Recalling the form of the disturbance in (1), it follows that the flow is unstable if and only if any one or more of the eigenvalues $\nu$ has a negative imaginary part. For a complete stability analysis the characteristic values of $\nu$ must be examined for all values of the vector $\mathbf{k}=\left[k_{x}, k_{y}\right]$.

3. Determination of the instabilities. The nature of the eigenvalues will now be determined. Make the substitutions

$$
\begin{gathered}
\xi=\nu+k_{*} \bar{U}_{a} \quad \eta=\nu+k_{*} \bar{U}_{b}-\frac{\rho_{*} \beta_{k}}{K-\rho_{*} \beta_{k}}\left[k_{*}\left(\bar{U}_{a}-\bar{U}_{b}\right)\right], \\
\eta_{0}^{2}=\frac{K \rho_{*} \beta_{k}}{\left(K-\rho_{*} \beta_{k}\right)^{2}}\left[k_{*}\left(\bar{U}_{a}-\bar{U}_{b}\right)\right]^{2}+\frac{\left(1-\rho_{*}\right)+\sigma_{k}}{K-\rho_{*} \beta_{k}}
\end{gathered}
$$

into the eigenvalue Eq. (4) and accompanying condition (5). This yields

$$
\eta^{2}+\frac{\rho_{*}}{K-\rho_{*} \beta_{k}} \xi^{2}\left(1-\frac{2 \beta_{k}}{\xi^{2}}\right)^{1 / 2}=\eta_{0}^{2}
$$

and

$$
\operatorname{Re}\left(1-2 \beta_{k} / \xi^{2}\right)^{1 / 2} \geq \beta_{k},
$$

which, in conjunction with the auxiliary relationship

$$
\xi-\eta=\left(K /\left(K-\rho_{*} \beta_{k}\right)\right)\left[k_{*}\left(\bar{U}_{a}-\bar{U}_{b}\right)\right],
$$

is equivalent to the eigenvalue problem. Because of the condition (9) this problem is nonalgebraic. Therefore, it is advantageous to construct a parent algebraic system possessing the eigenvalue problem as a subsystem. If all restrictions on $\operatorname{Re}\left(1-\left(2 \beta_{k} / \xi^{2}\right)\right)^{1 / 2}$ are removed then Eq. (8), together with auxiliary Eq. (10), is equivalent to a fourth 
degree polynomial in $\nu$ and this is taken as the parent system. Let us distinguish two branches of the parent system, calling our eigenvalue problem the principal or $P$-branch and the remainder of the system, where $\operatorname{Re}\left(1-\left(2 \beta_{k} / \xi^{2}\right)\right)^{1 / 2}<\beta_{k}$, the subsidiary or $S$-branch. In mathematical form

$$
\begin{aligned}
& P \text {-branch }\left\{\begin{array}{c}
\eta^{2}+\frac{\rho_{*}}{K-\rho_{*} \beta_{k}} \xi^{2}\left(1-\frac{2 \beta_{k}}{\xi^{2}}\right)^{1 / 2}=\eta_{0}^{2}, \\
\operatorname{Re}\left(1-\frac{2 \beta_{k}}{\xi^{2}}\right)^{1 / 2} \geq \beta_{k},
\end{array}\right. \\
& \begin{array}{c}
\text { S-branch } \\
\text { Part 1 } \\
S_{1}
\end{array} \\
& \begin{array}{c}
\eta^{2}+\frac{\rho_{*}}{K-\rho_{*} \beta_{k}} \xi^{2}\left(1-\frac{2 \beta_{k}}{\xi^{2}}\right)^{1 / 2}=\eta_{0}^{2}, \\
0<\operatorname{Re}\left(1-\frac{2 \beta_{k}}{\xi^{2}}\right)^{1 / 2}<\beta_{k},
\end{array} \\
& \begin{array}{c}
\text { Part 2 } \\
S_{2}
\end{array}
\end{aligned}\left\{\begin{array}{c}
\eta^{2}-\frac{\rho_{*}}{K-\rho_{*} \beta_{k}} \xi^{2}\left(1-\frac{2 \beta_{k}}{\xi^{2}}\right)^{1 / 2}=\eta_{0}^{2}, \\
\operatorname{Re}\left(1-\frac{2 \beta_{k}}{\xi^{2}}\right)^{1 / 2} \geq 0 .
\end{array}\right.
$$

The auxiliary Eq. (10) must be satisfied simultaneously with each of the above basic branch equations and conditions. It follows now that the number of roots of the eigenvalue problem is four or less.

The real loci of the basic branch equations are plotted in the $(\xi, \eta)$-plane for the case $K>\rho_{*} \beta_{k}$. These loci are shown in Fig. 2, where

$$
\begin{aligned}
& \xi_{s}^{2}=\frac{2 \beta_{k}}{K^{2}} \quad \eta_{s}^{2}=\eta_{0}^{2}-\frac{\rho_{*} \beta_{k}}{K-\rho_{*} \beta_{k}}\left(\frac{2 \beta_{k}}{K^{2}}\right), \\
& \xi_{0}^{2}=\beta_{k}+\left[\beta_{k}^{2}+\frac{\left(K-\rho_{*} \beta_{k}\right)^{2}}{\rho_{*}^{2}} \eta_{0}^{4}\right]^{1 / 2} .
\end{aligned}
$$

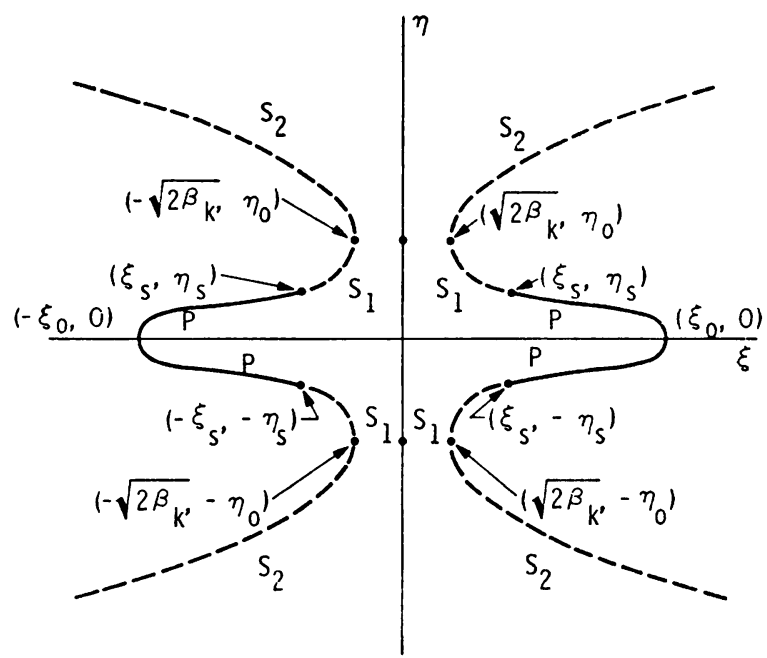

FIG. 2. The real locus of the basic branch equations. 


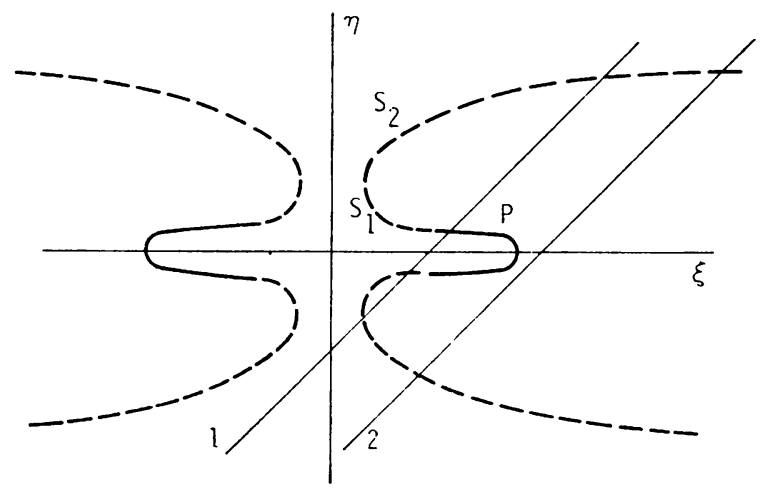

FIG. 3. Real intersections of the auxiliary equation with the branch equations

In Fig. 2 the solid lines represent the $P$-branch and the broken lines the $S_{1}$ and $S_{2}$ branches. Symmetry occurs about both the $\xi$ and $\eta$-axes. Tangents to the curve are vertical at the points $\left(\left(2 \beta_{k}\right)^{1 / 2}, \eta_{0}\right)$ and $\left(\xi_{0}, 0\right)$. Also, it is very significant that, except at the two points $\left(0, \pm \eta_{0}\right)$, there is no real locus for $|\xi|<\left(2 \beta_{k}\right)^{1 / 2}$.

The real locus of the auxiliary Eqs. (10) is a straight line having a slope of 1, as shown by two examples (lines 1 and 2 ) in Fig. 3 . Intersections in the real $(\xi, \eta)$-plane of this line with the locus of the basic branch equations represent real roots of the parent algebraic system. Location of these intersections indicates the particular branch to which each real root belongs. For situations where there are four real intersections the eigenvalue problem represented by the $P$-branch can have no complex roots and the system is stable. Referring now to Fig. 4, let four lines be drawn in the real $(\xi, \eta)$-plane with a slope of 1 . Line 1 passes through the origin while lines $1^{\prime}, 2$ and $2^{\prime}$ are drawn tangent to the locus of the branch equations. Analytical examination of the loci reveals that under the conditions

$$
\frac{\rho_{*}}{K-\rho_{*} \beta_{k}}<1 \text { and } \frac{\rho_{*}}{K-\rho_{*} \beta_{k}}<-\left(\frac{3}{64}\right)^{1 / 2}+\left[\frac{3}{64}+2\left(\frac{\eta_{0}}{\left.2 \beta_{k}\right)^{1 / 2}}\right)^{2}\right]^{1 / 2}
$$

auxiliary lines falling between 2 and $2^{\prime}$ of Fig. 4 will always have four real intersections while all auxiliary lines to the right of $2^{\prime}$ will have two real intersections. If additionally

$$
\left(\frac{\rho_{*}}{K-\rho_{*} \beta_{k}}\right)^{2}>4\left(\frac{\eta_{0}}{\left(2 \beta_{k}\right)^{1 / 2}}\right)^{2}\left[1-\left(\frac{\eta_{0}}{\left(2 \beta_{k}\right)^{1 / 2}}\right)^{2}\right]
$$

then auxiliary lines occur between 1 and $1^{\prime}$ with four real intersections while auxiliary lines between $1^{\prime}$ and 2 have 2 real intersections. Thus it is possible to discern two regions corresponding to situations of definitely stable flow. These regions are stability zone 1 between lines 1 and $1^{\prime}$ on Fig. 4 and stability zone 2 between lines 2 and $2^{\prime}$.

For a given fluid system in a particular stationary state, the sufficient conditions (17) and (18) become a requirement on the range of $k$. In the case of normal gases and liquids $\rho_{*}$ and $\beta$ are extremely small, and consequently the above conditions, as well as the earlier assumption of $K>\rho_{*} \beta_{k}$, are satisfied for all disturbances except those of extremely long length.

At this point it is helpful to examine the behavior of the equations and resulting 
curves in the $(\xi, \eta)$-plane for a fluid system of fixed properties, under the influence of a particular disturbance, as the velocity difference $\left(U_{a}-U_{b}\right)$ alone increases. Since $\left(U_{a}-U_{b}\right)$ and $k_{*}$ only appear together it is convenient to define the dimensionless velocity $\bar{U}=k_{*}\left(\bar{U}_{a}-\bar{U}_{b}\right)$. Now as the velocity difference, or $\bar{U}$, increases, the $\xi$ and $\eta$ intercepts of the auxiliary line move rightward and downward respectively. At the same time the real locus of the branch equations enlarges. However, close examination shows that for normal gases and liquids transition of the line from an initial position through the origin, as corresponds to a velocity difference of zero, to a location at the right of $\left(\xi_{0}, 0\right)$ corresponds to a nearly imperceptible change in the location of the locus of the branch equations. Thus, as $\bar{U}$ alone increases, the fluid system experiences consecutively conditions of definite stability (stability zone 1), possible instability (between stability zones 1 and 2), definite stability (stability zone 2) and finally possible instability (to the right of stability zone 2).

Consider now the question of stability in the region between stability zones 1 and 2 . In this region the parent system possesses a conjugate pair of complex roots and instability or stability depends on whether or not this pair belongs to the $P$-branch. An exceptional situation of known stability is noted when the auxiliary line passes through the point $\left(0,-\eta_{0}\right)$ which corresponds to a real root of multiplicity two. The branch to which a particular root belongs depends on the nature of the real part of $\left(1-\left(2 \beta_{k} / \xi^{2}\right)\right)^{1 / 2}$, and information of this nature must follow in accordance with the branch equations and the root in question. Thus, since $\left(K-\rho_{*} \beta_{k}\right) / \rho_{*}$ is real and positive (considering $K>\rho_{*} \beta_{k}$ ), it follows according to the branch relations (11) through (16) that a root belongs to the $S_{2}$-branch if $\operatorname{Re}\left(\eta_{0}^{2}-\eta^{2}\right) / \xi^{2} \leq 0$, to the $S_{1}$-branch if $0<$ $\operatorname{Re}\left(\eta_{0}^{2}-\eta^{2}\right) / \xi^{2}<\rho_{*} \beta_{k} /\left(K-\rho_{*} \beta_{k}\right)$, and to the $P$-branch if

$$
\operatorname{Re}\left(\eta_{0}^{2}-\eta^{2}\right) / \xi^{2} \geq \rho_{*} \beta_{k} /\left(K-\rho_{*} \beta_{k}\right)
$$

with the above being evaluated by substitution of the root in question.

For fixed values of all physical parameters, including $\bar{U}$, it is noted that $\left(\eta_{0}^{2}-\eta^{2}\right) / \xi^{2}$ is real and positive along the real locus of the combined $S_{1}$ and $P$-branches in the fourth quadrant. Further, it follows from the branch equations (11) and (13) that $\left(\eta_{0}^{2}-\eta^{2}\right) / \xi^{2}$ has a value, at any given point on this locus, that is greater than its value at all other points to its left (lower values of $\xi$ ) along the locus. Now let $\bar{U}_{T}$ be that value of $\bar{U}$

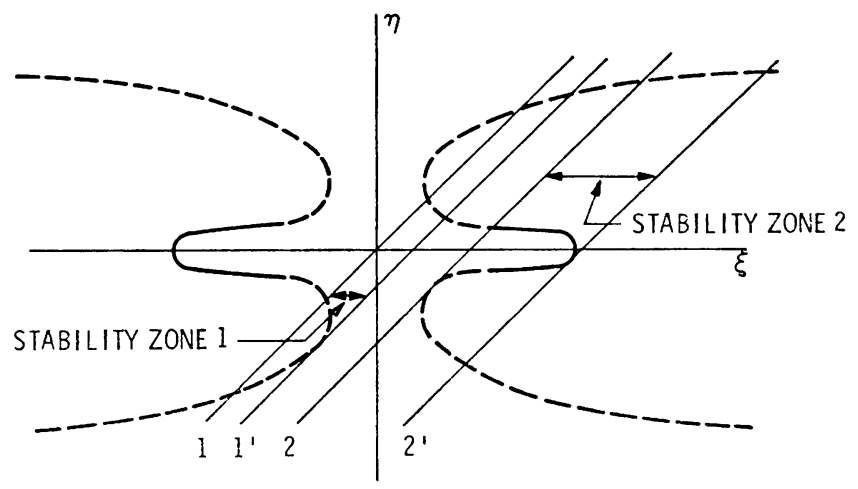

FIG. 4. Stability zones in the real $(\xi, \eta)$-plane 


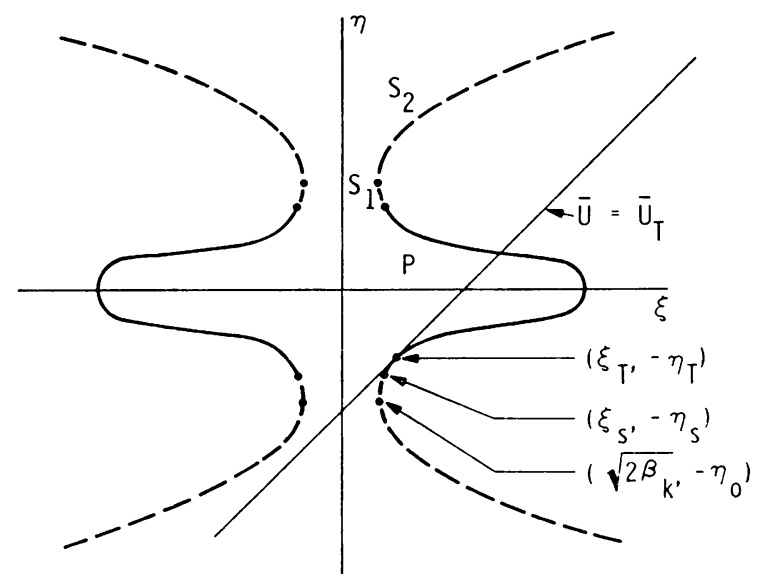

FIG. 5. The boundary of stability zone 2 at $\bar{U}=\bar{U}_{T}$

for which the system first enters stability zone 2 with the corresponding real root of multiplicity two being $\left(\xi_{T},-\eta_{T}\right)$. Suppose $\xi_{T}=\xi_{S}+a$, where $a$ is some positive real number, with the point $\left(\xi_{T},-\eta_{T}\right)$ consequently being on the $P$-branch as shown in Fig. 5.

According to the above discussion and assumptions it follows that

$$
\left(\eta_{0}^{2}-\left(-\eta_{T}\right)^{2}\right) / \xi_{T}^{2}=\rho_{*} \beta_{k} /\left(K-\rho_{*} \beta_{k}\right)+b
$$

where $b$ is a positive real number. For $\bar{U}<\bar{U}_{T}$, the auxiliary line is in the region between stability zones with a corresponding conjugate pair of complex roots that are destined to become the multiple root $\left(\xi_{T},-\eta_{T}\right)$ when $\bar{U}=\bar{U}_{T}$. In conjuction with these complex roots

$$
\frac{\eta_{0}^{2}-\eta^{2}}{\xi^{2}}=\frac{\eta_{0}^{2}-\left(-\eta_{T}\right)^{2}}{\xi_{T}^{2}}-\epsilon=\frac{\rho_{*} \beta_{k}}{K-\rho_{*} \beta_{k}}+(b-\epsilon)
$$

and according to Eq. (19) the roots are zeros of the $P$-equation if

$$
\operatorname{Re} \epsilon \leq b \text {. }
$$

Since the pair of complex roots are always zeros of the parent algebraic system, they must be continuous functions of the coefficients of the parent equation (see, e.g., Marden [2, pp. 3-4]). These coefficients are in turn continuous functions of $\bar{U}$ and thus the conjugate pair of complex roots, as well as the term $\eta_{0}^{2}-\eta^{2} / \xi^{2}(\xi \neq 0$ in the neighborhood of $\xi_{T}$ ), must vary continuously with $\bar{U}$. Thus $\operatorname{Re} \epsilon$ is made arbitrarily small by choosing $\bar{U}$ arbitrarily close to $\bar{U}_{T}$. As a result there must exist a region, immediately before stability zone 2, where $\operatorname{Re} \epsilon<b_{T}$ and the pair of complex roots belongs to the $P$-branch. This must be a zone of unstable flow, and by similar reasoning there must be another unstable region just after stability zone 2 . While the condition $\xi_{T}>\xi_{s}$ is necessary and sufficient for instability in the neighborhood of the inner boundary of stability zone 2 , it is noted that the alternative condition $\xi_{T}<\xi_{8}$ is not sufficient to disallow instability throughout the region between stability zones 1 and 2 . The degree to which the imaginary part of the complex roots may become large is influenced by the width of the 
region between stability zones 1 and 2 , and therefore this width, or the $\xi$-coordinate value $\left(2 \beta_{k}\right)^{1 / 2}$, is an indication of the rate of growth of the corresponding instabilities.

It should be noted that continuity of the roots requires that the coefficient of the highest degree term of the parent polynomial be different from zero. This condition is satisfied for all disturbances except those nearly infinite length disturbances having the specific wave number $k=\rho_{*} \beta /\left(1-\rho_{*}^{2}\right)$.

Physical conditions corresponding to $\xi_{T}>\xi_{\text {s }}$ will now be determined. Knowing $\xi_{s} \cong\left(2 \beta_{k}\right)^{1 / 2}$ for normal gases, it is equivalent and much more expedient to determine conditions such that $d \eta / d \xi>1$ at $\left(\xi_{s},-\eta_{s}\right)$, the end point of the $P$-branch in the fourth quadrant, for the velocity $\bar{U}=\bar{U}_{s}$ locating the auxiliary line through $\left(\xi_{s},-\eta_{s}\right) . \bar{U}$, is unique and given by the relationship

$$
\bar{U}_{s}=\frac{\left(2 \beta_{k}\right)^{1 / 2}}{K}+\left[\frac{1}{K}\left[\left(1-\rho_{*}\right)+\sigma_{k}\right]\right]^{1 / 2} .
$$

For this velocity the requirement that $d \eta / d \xi>1$ at $\left(\xi_{s},-\eta_{s}\right)$ reduces to

$$
\left(4-3 K^{2}\right)>\frac{2 \beta_{k} K}{\rho_{*}^{2}}\left[\left(1-\rho_{*}\right)+\sigma_{k}\right]+\frac{4 \beta_{k}^{2}}{\rho_{*}}\left[\frac{2 \beta_{k}}{K}\left(\left(1-\rho_{*}\right)+\sigma_{k}\right)\right]^{1 / 2}
$$

which is a sufficient condition for instability prior to stability zone 2 . Henceforth these instabilities will be referred to as the initial instabilities. Examination of the condition (20) shows that for normal fluids (small $\rho_{*}$ and $\beta$ ) the initial instabilities must occur over at least a limited range of $k$. Of course failure to be in this range does not necessarily disallow initial instabilities.

The value of $\bar{U}$ marking the occurrence of the initial instabilities will now be determined. A readily accessible and accurate approximation for this velocity $\bar{U}$ is the value of $\bar{U}_{i}$ which locates the auxiliary line through the point $\left(0,-\eta_{0}\right)$ as shown in Fig. 6.

From the equations for $\eta_{0}$ and the auxiliary line it follows that

$$
\bar{U}_{i}=\left[\frac{1}{K}\left[\left(1-\rho_{*}\right)+\sigma_{k}\right]\right]^{1 / 2}
$$

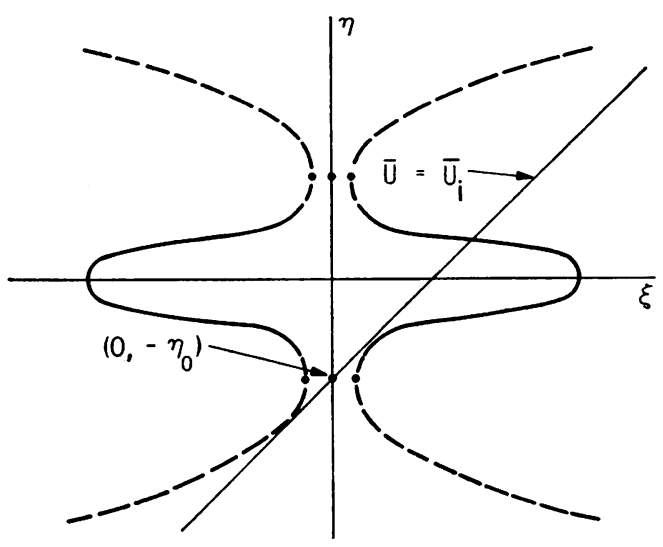

FIG. 6. The velocity of initial instabilities, approximated by $\bar{U}_{i}$ 


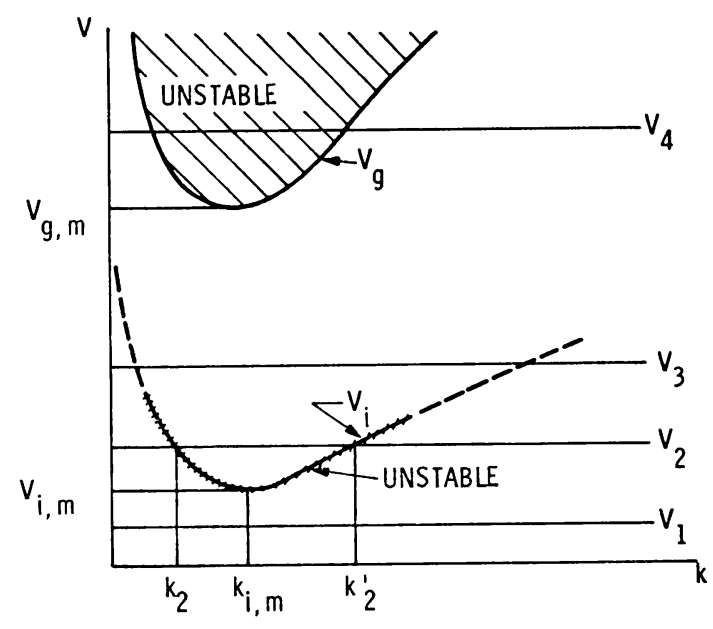

FIg. 7. Typical instabilities for a gas over a liquid

or in dimensional terms

$$
\left[k_{*}\left(U_{a}-U_{b}\right)\right]_{i}=\left[\frac{g\left(1-\rho_{*}\right)}{k}+\frac{T_{s}}{\rho_{b}} k\right]^{1 / 2} .
$$

For normal gases $\beta$ is very small, thus making the points $\left(-\left(2 \beta_{k}\right)^{1 / 2},-\eta_{0}\right)$ and $\left(\left(2 \beta_{k}\right)^{1 / 2},-\eta_{0}\right)$ extremely close to each other for all but very small $k$. In such cases $\bar{U}_{i}$ for all practical purposes exactly marks the occurrence of the initial instabilities.

Instabilities also occur in the neighborhood of the outer boundary (line $2^{\prime}$ in Fig. 4) of stability zone 2. This instability is followed at higher $\bar{U}$ by an unlimited region of unknown stability conditions. This region is assumed to be unstable. Such an assumption is suggested by the nature of results usually obtained in analytic stability analyses as well as results obtained in experimental investigations. Grounds for the assumption will become even more sound when viewed in light of the relationship of this analysis to the classical problem of Kelvin. The instabilities following stability zone 2 will be referred to as the gross instabilities.

The onset of the gross instabilities will be represented by the velocity $\bar{U}=\bar{U}_{\text {。 }}$ and for resaons which become obvious later, $\bar{U}_{\diamond}$ is excellently approximated by Kelvin's solution written as

$$
\bar{U}_{K}=\left(\frac{1+\rho_{*}}{\rho_{*}}\right)^{1 / 2}\left[\frac{1}{K}\left[\left(1-\rho_{*}\right)+\sigma_{k}\right]\right]^{1 / 2}=\left(\frac{1+\rho_{*}}{\rho_{*}}\right)^{1 / 2} \bar{U}_{i}
$$

or in dimensional terms

$$
\left[k_{*}\left(U_{a}-U_{\iota}\right)\right]_{K}=\left(\frac{1+\rho_{*}}{\rho_{*}}\right)^{1 / 2}\left[\frac{g\left(1-\rho_{*}\right)}{k}+\frac{T_{s}}{\rho_{b}} k\right]=\left(\frac{1+\rho_{*}}{\rho_{*}}\right)^{1 / 2}\left[k_{*}\left(U_{a}-U_{b}\right)\right]_{i} .
$$

For a gas over a liquid, the shape of the $P$-branch is long and thin and so another good approximation for $\bar{U}_{g}$ is that value of $\bar{U}$ which passes the auxiliary line through the point $\left(\xi_{0}, 0\right)$. Determination of this velocity involves the solutions of a quadratic equation. Although usually unnecessary, greater accuracy can be obtained by a graphical 
iteration procedure involving determination of improved velocity approximations from auxiliary lines drawn tangent to the $P$-branch plotted on the basis of less accurate values of $\bar{U}$.

It is now possible to assess the behavior of a given fluid system under the influence of a general disturbance. In so doing, the regions of stability and instability are plotted in a plane of velocity difference $v=k_{*}\left[U_{a}-U_{b}\right]$ versus wave number, as shown in Fig. 7. The curve for $\left[k_{*}\left(U_{a}-U_{b}\right)\right]_{i}$ follows from Eq. (22) with the broken portion representing the region where the condition (20) is not satisfied and where instability is therefore uncertain. Dashes along the solid portion illustrate the fact that initial instabilities occur in a narrow band of $\bar{U}$ for each value of $k$. The curve for $\left[k_{*}\left(U_{a}-U_{b}\right)\right]_{o}$ corresponds to the boundary of stability zone 2. According to Fig. 7, a fluid system is expected to be stable at low velocities. Instabilities first occur at the velocity $\left[k_{*}\left(U_{a}-U_{b}\right)\right]_{i, m}$, determined from Eq. (22) by elementary calculus to be

$$
\left[k_{*}\left(U_{a}-U_{b}\right)\right]_{i, m}^{2}=2\left[g\left(\rho_{b}-\rho_{a}\right) T \cdot / \rho_{b}^{2}\right]^{1 / 2}
$$

and have a wave number $k_{i, m}$ given by the relationship

$$
k_{i, m}=\left[g\left(\rho_{b}-\rho_{a}\right) / T_{s}\right]^{1 / 2} .
$$

At velocities just above this value the system is unstable, as indicated by position 1 in Fig. 7. Instabilities at such a point, however, are of a weak, selective nature, as they are confined to two narrow bands of wave numbers located about the values $k_{1}$ and $k_{1}^{\prime}$. At a velocity $\left[k_{*}\left(U_{a}-U_{b}\right)\right]_{,, m}$ an additional instability is initiated. This instability is of a much stronger nature, being composed at any given velocity of a continuous spectrum of unstable waves as illustrated by position 2 of Fig. 7 . Finally it is pointed out that the tendency to become unstable increases as the disturbance direction rotates toward that of the stationary state velocity. This conclusion is indicated by the occurrence of $k_{*}$ as a coefficient of $\left(U_{a}-U_{b}\right)$ in Fig. 7, and it testifies to the validity of Yih's [6] generalization of Squire's theorem.

To complete the above description the velocity of propagation $\gamma$ is determined for the initial waves. From the real $(\xi, \eta)$-plot it follows, at least to within very close accuracy for normal fluids, that for the initial instabilities $\eta=-\eta_{0}$. Substituting the definitions of $\eta$ and $\eta_{0}$ and rearranging, with the realization that $\gamma=-n / k$, yields

$$
\begin{aligned}
\gamma=k_{*} U_{b}-\frac{\rho_{*} \beta_{k}}{K-\rho_{*} \beta_{k}}\left[k_{*}\left(U_{a}-U_{b}\right)\right] \\
+\left[\frac{K \rho_{*} \beta_{k}}{\left(K-\rho_{*} \beta_{k}\right)^{2}}\left[k_{*}\left(U_{a}-U_{b}\right)\right]^{2}+\frac{g\left[\left(1-\rho_{*}\right)+\sigma_{k}\right]}{k-\frac{\rho_{*} \beta}{2}}\right]^{*} .
\end{aligned}
$$

Taking advantage of the smallness of $\rho_{*}$ and $\beta$, it follows that

$$
\gamma \cong k_{*} U_{b}+\left(\frac{g \lambda}{2 \pi}+\frac{2 \pi T_{b}}{\lambda \rho_{b}}\right)^{1 / 2}
$$

The right-hand side may be recognized as the classical result for the propagation speed of surface waves on a liquid of infinite depth. It is also of interest to note the relationship of this propagation speed to the accompanying wind velocity. Again from the real $(\zeta, \eta)$-plot, it is true within close accuracy that for initial instabilities $\xi=0$. Substituting the def- 


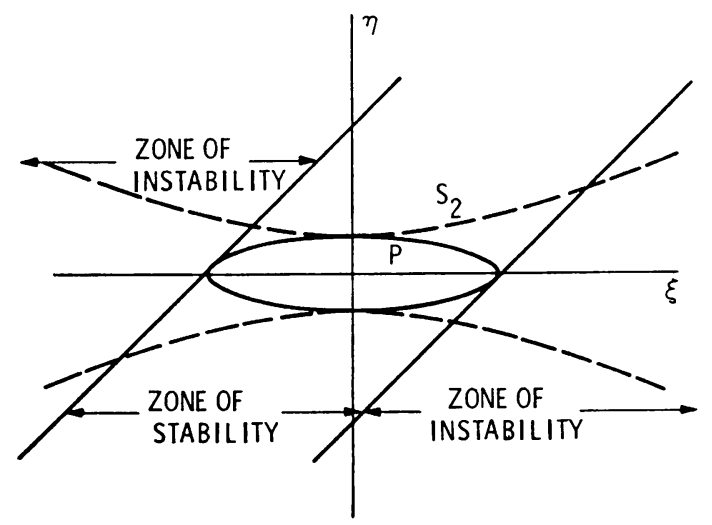

FIG. 8. Stability zones in the real $(\xi, \eta)$-plane for $\beta=0$

inition of $\zeta$ into this relationship and rearranging yields $\left[k_{*}\left(U_{a}-H_{b}\right)\right]_{i}=\gamma-k_{*} U_{b}$. Thus wind and wave travel together.

4. Kelvin's solution as a special case. Suppose the heterogeneity of the gas is ignored. In this case $\beta=0$ and the problem reduces to that considered by Kelvin. Examination of the real $(\xi, \eta)$-locus of the branches when $\beta=0$ reveals the following values for the characteristic dimensions and coordinates:

$$
\begin{aligned}
\left(2 \beta_{k}\right)^{1 / 2} & =0 & \eta_{0}^{2} & =\left[\left(1-\rho_{*}\right)+\sigma_{k}\right], \\
\xi_{s}^{2} & =0 & \eta_{s}^{2} & =\eta_{0}^{2} .
\end{aligned}
$$

The most significant feature of these results is the location of the point $\left(\left(2 \beta_{k}\right)^{1 / 2}, \eta_{0}\right)$ on the $\eta$-axis. As a result the initial instabilities do not appear in Kelvin's problem. According to the branch equations the $S_{1}$-branch completely degenerates while the $P$-branch becomes an ellipse and the $S_{2}$-branch a hyperbola. Each of the latter two branches is now separately equivalent to a second degree polynomial and thus the stability zones must be as shown on Fig. 8.

The equations of the branches are now independent of $\bar{U}$. Bounds on stability are thus easily obtained mathematically by determining the point along the $P$-branch where $d \eta / d \xi=1$, passing the auxiliary line through the point, and then solving the resulting equation for $\bar{U}$. The resulting velocity is

$$
\left(U_{a}-U_{b}\right)^{2}=\frac{g\left(\rho_{a}+\rho_{b}\right) k}{\rho_{a} \rho_{b} k_{x}^{2}}\left[\left(\rho_{b}-\rho_{a}\right)+T_{s} k^{2} / g\right] .
$$

This is the critical velocity found by Kelvin. The use of Kelvin's solution as an approximation to the gross instabilities for $\beta \neq 0$ may now be appreciated.

5. Numerical results for air over water. As a particular example consider air blowing over sea water and assume

$$
\begin{aligned}
\rho_{b} & =1.02 \mathrm{gm} / \mathrm{cm}^{3} & \rho_{*} & =1.26 \times 10^{-3}, \\
T_{\imath} & =74 \text { dynes } / \mathrm{cm} & \beta & =1.1 \times 10^{-6} \mathrm{~cm}^{-1} .
\end{aligned}
$$


Calculations according to Eq. (22) for the initial instabilities and use of Kelvin's solution as an approximation (found extremely accurate in this case) to the boundary of the gross instability region results in the values shown in Fig. 9. The range of the solid portion of the initial instability curve extends from $k=0.75 \mathrm{~cm}^{-1}$ to $k=18.75 \mathrm{~cm}^{-1}$. The velocity difference at which instability first occurs is calculated according to Eq. (23) and found to be $23.1 \mathrm{~cm} / \mathrm{sec}$, while the corresponding wavelength is calculated as $\lambda_{i, m}=$ $2 \pi / k_{i, m}=1.71 \mathrm{~cm}$. As a typical example the real $(\xi, \eta)$-locus of the branches is plotted in Fig. 10 for $k=3.67 \mathrm{~cm}^{-1}$. Because changes in the locus due to changes in $\bar{U}$ are very slight, Fig. 10 may be applied to all low velocity differences.

As mentioned above, some indication of the rate of growth of initial instabilities is given by the value of $\left(2 \beta_{k}\right)^{1 / 2}$. Thus, for the initial instability at $k=3.67 \mathrm{~cm}^{-1}$ it is expected that

$$
\operatorname{Im} n \cong(k g)^{1 / 2} \operatorname{Im} \xi \sim(k g)^{1 / 2}\left(2 \beta_{k}\right)^{1 / 2}=.033 \sec ^{-1}
$$

and as the imaginary part of $n$ (see Eq. (1)) characterizes the growth rate, it is indicated that for air and water the initial instabilities grow slowly.

6. Concluding Remarks. Density stratification in a gas flowing above a liquid has been found to have a destabilizing influence taking the form of a relatively weak

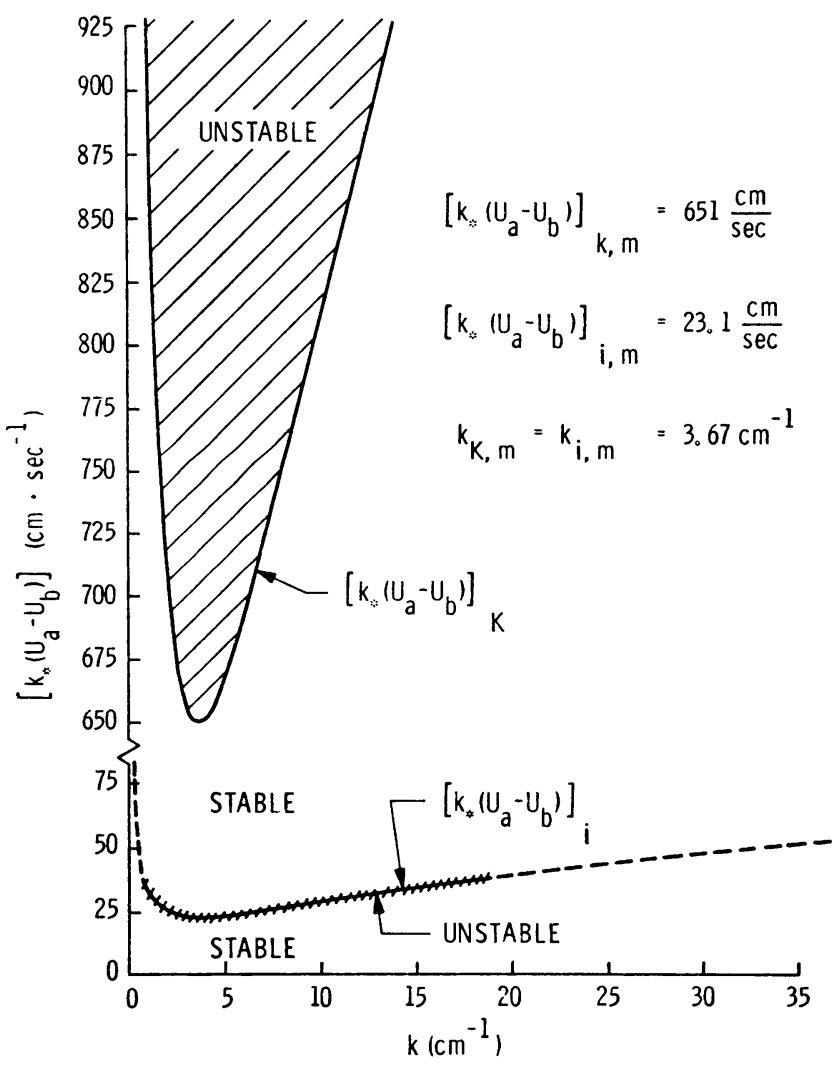

FIG. 9. Instabilities for air blowing over water 


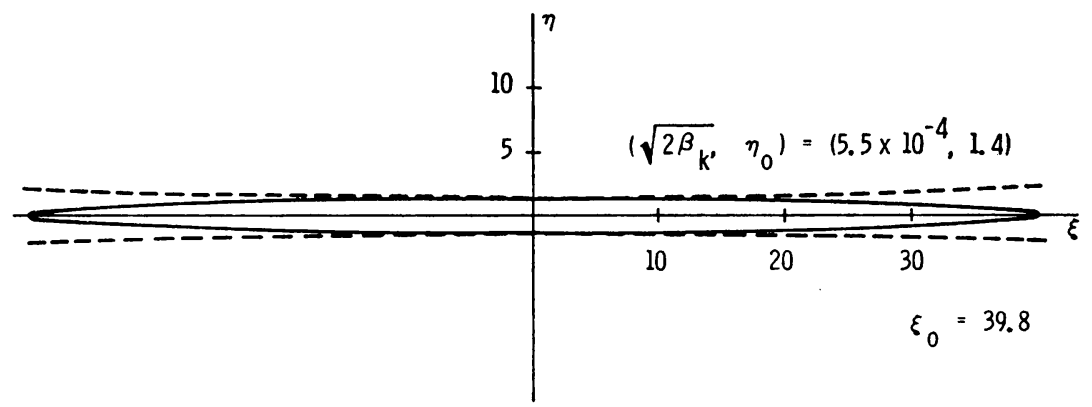

FIG. 10. The real $(\xi, \eta)$-locus of the branches for air over water at low velocity differences and $k=3.67 \mathrm{~cm}^{-1}$

initial instability preceding the stronger classical instability of Kelvin. The qualitative nature of this initial instability, as illustrated by the lower curve of Fig. 9, is in agreement with experimental observation. Numerical results for air and water also appear reasonably in accord with experimental values of the critical velocity and wave number at which instability first occurs. However, the analysis indicates that the rate of growth of the initial instabilities, as well as the width of the two bands of unstable wave numbers comprising this instability at a given velocity, decreases with the factor $\beta$, and as a result, growth rates for air and water are likely to be quite small. The numerical estimate made herein for the growth rates may be somewhat lower than actual values since it is based on density stratification in an idealized atmosphere. For an actual atmosphere density gradients, and therefore growth rates, could be larger due to the added effect of temperature variations. Along these lines it is noted that the initial instability may be especially pertinent to situations where the liquid is at a much lower temperature than the gas, thus causing steep density gradients to be set up in the gas near the liquid.

Regarding the nature of the initial instability, the following physical mechanism is suggested.

An interaction occurs between an internal wave in the stratified upper fluid and a capillary-gravity wave in the lower one. The wave length and phase speed of the two waves are such that the waves are amplified. That this occurs is indicated by the eigenvalue calculation which shows the propagation speed and wave length to be related as in the classical form of capillary-gravity waves. Since the stratification is weak and the internal wave travels very slowly relative to the upper fluid and because wind and wave travel together the internal wave can match up and resonate with the capillarygravity wave.

\section{REFERENCES}

[1] S. Chandrasekhar, Hydrodynamic and hydromagnetic stability, Oxford Univ. Press, New York, 1961 [2] M. Marden, The geometry of the zeros, Math. Surveys, no. 3, Amer. Math. Soc., Providence, R. I., 1949

[3] W. H. Munk, A critical wind speed for air-sea boundary processes, J. Marine Research 6, 203 (1947)

[4] S. Ostrach and A. Koestel, Film instabilities in two-phase flows, 6th National Heat Transfer Conference ASME, Boston, Mass., August 1963

[5] J. F. Sontowski, The stability of flow of a gas over a liquid, Ph.D. Thesis, University of Delaware, 1966

[6] C. S. Yih, Stability of two-dimensional parallel flows for three-dimensional disturbances, Quart. Appl. Math. 12, 434 (1955) 